\title{
Differential Cyclic AMP Dependence of Facilitation at Aplysia Sensorimotor Synapses as a Function of Prior Stimulation: Augmentation versus Restoration of Transmitter Release
}

\author{
Marc Klein \\ Clinical Research Institute of Montreal, Montreal, Quebec H2W 1R7, Canada
}

Synaptic facilitation at sensory-to-motor neuron synapses in Aplysia is a mechanism contributing to a simple form of learning called behavioral sensitization. Previous work has shown that facilitation is mediated in part by the neurotransmitter 5-HT acting through CAMP to broaden presynaptic action potentials and thus increase transmitter release from the sensory neuron terminals. Other studies have indicated that 5-HT causes facilitation by more than one mechanism, depending on whether the synapse has first been depressed by prior stimulation. The present study examines the involvement of CAMP in facilitation at depressed synapses by utilizing the adenylyl cyclase activator $7 \beta$-desacetyl-7 $\beta-[\Gamma-(N-$ methylpiperazino)-butyryl] forskolin (7B-forskolin) and 5-HT separately and in combination. Facilitation at relatively rested synapses can be mimicked with application of $7 \mathrm{~B}$-forskolin, whereas transmission at synapses that have been subjected to repeated stimulation is not affected. The forskolin derivative by itself increases cAMP levels in sensory neurons and potentiates 5-HT-induced stimulation of CAMP 2-10-fold. Nonetheless, joint application of 7B-forskolin and 5-HT at submaximal concentrations to depressed synapses causes the same amount of facilitation as $5-\mathrm{HT}$ alone. This finding implies that facilitation by 5-HT at repeatedly stimulated synapses is not mediated by CAMP alone. In addition, facilitation by $7 B$-forskolin at relatively rested synapses can occur without prolongation of action potentials, suggesting that CAMP can act in more than one way to enhance transmission. Taken together with earlier findings, the present results suggest that at least three distinct processes participate in facilitation by $5-\mathrm{HT}$.

[Key words: learning, neuromodulation, Aplysia, 5-HT, CAMP, forskolin, facilitation, transmitter release, sensitization, dishabituation, synapses, cultured neurons]

Activation of various second-messenger systems by neurotransmitters has been implicated in a number of forms of synaptic plasticity that are thought to be involved in the behavioral changes that result from learning. For example, in neuronal systems including the mammalian hippocampus, molluscan ganglia, and crustacean neuromuscular junctions, several pro-

\footnotetext{
Received Sep. 11, 1992; revised Feb. 26, 1993; accepted Mar. 18, 1993.

I thank T. W. Abrams, V. F. Castellucci, and L.-E. Trudeau for comments on an earlier version of the manuscript. This work was supported by NIMH Grant MH45397 and by funding from the Richard and Edith Strauss Canada Foundation.

Correspondence should be addressed to Marc Klein, Clinical Research Institute of Montreal, 110 Pine Avenue West, Montreal, Quebec H2W 1R7, Canada.

Copyright (C) 1993 Society for Neuroscience 0270-6474/93/133793-09\$05.00/0
}

tein kinases (Castellucci et al., 1980, 1982; Schacher et al., 1988; Dixon and Atwood, 1989a,c; Malenka et al., 1989; Malinow et al., 1989; Braha et al., 1990; Matzel et al., 1990; Sacktor and Schwartz, 1990; Etcheberrigaray et al., 1992; Silva et al., 1992a,b; Sugita et al., 1992), the inositol trisphosphate system (Dixon and Atwood, 1989b,c), and the NO synthase pathway (O'Dell et al., 1991; Schuman and Madison, 1991) have been shown to play a role in the synaptic changes induced by different kinds of nerve stimulation. Neuromodulation at the sensory neuron synapses of the marine mollusk Aplysia californica has been proposed to contribute to nonassociative as well as to associative learning in this animal. Habituation, dishabituation, and sensitization of defensive withdrawal reflexes of Aplysia are nonassociative forms of behavioral plasticity that are accompanied by appropriate changes in transmission at these synapses (Castellucci and Kandel, 1974, 1976). Evidence has also been presented that classical conditioning of the defensive withdrawal reflexes may involve an activity-dependent amplification of mechanisms underlying behavioral sensitization (Hawkins et al., 1983; Walters and Byrne, 1983; Abrams et al., 1991).

In Aplysia, electrical stimulation of pathways that mediate sensitization and dishabituation leads to an increase in transmitter release from sensory neurons in the defensive withdrawal pathways, and this synaptic facilitation has been proposed to contribute to the behavioral change. Earlier work showed that release of 5-HT and other transmitters from facilitator interneurons is responsible for the facilitation and that activation by these transmitters of a neurotransmitter-sensitive adenylyl cyclase in the membrane of the sensory neurons leads to an increase in intracellular levels of cAMP that, in turn, contributes to enhanced transmitter release (Brunelli et al., 1976; Bernier et al., 1982; Ocorr and Byrne, 1985; Glanzman et al., 1989).

The model for the modulation of transmitter release by cAMP (Klein and Kandel, 1980; Klein et al., 1980; Hochner et al., 1986a) includes the following steps: (1) cAMP causes phosphorylation of one or more type of membrane potassium channel or of molecules closely associated with the channels; (2) phosphorylation causes these channels to close, thereby slowing repolarization of the action potential in the sensory neurons; (3) the increase in duration of the action potential prolongs the entry of calcium into the presynaptic terminals; and (4) increased duration of calcium influx results in prolongation of transmitter release.

More recent work has shown that facilitation by 5-HT, rather than being a unitary process, is composed of at least two components, depending on the activation history of the synapse at which it is elicited (Hochner et al., 1986b; see also Gingrich and 
Byrne, 1985). At relatively rested synapses, artificial broadening of the action potential or of a presynaptic voltage-clamp depolarization leads to significant increases in the amount of transmitter released from the sensory neurons (Hochner et al., 1986a).

However, at synapses that have been activated repeatedly and that have consequently undergone synaptic depression-as presumably occurs in behavioral habituation-broadening of the presynaptic spike or voltage-clamp depolarization has little effect on transmitter release (Hochner et al., 1986b). The fact that 5-HT can nonetheless cause significant facilitation under these circumstances argues that a process other than action potential broadening is largely responsible for the facilitation that contributes to dishabituation. Further study has shown that facilitation by 5-HT at rested and at depressed synapses can also be distinguished pharmacologically, suggesting that the two sets of processes differ at the biochemical level as well (Braha et al., 1990).

The present study was designed to examine the role of the cAMP cascade in facilitation at rested as well as at depressed synapses by examining the relationship between synaptic facilitation in cell culture and cAMP levels in sensory neurons prepared in the same way as the cultured cells. The results indicate that while facilitation at relatively rested synapses, a process that will be called "5-HT-mediated synaptic augmentation"or simply "augmentation"-may involve mobilization of the cAMP pathway, facilitation at more extensively stimulated synapses-or synaptic "restoration"-appears to depend on other biochemical pathways. In addition, the results suggest that synaptic augmentation by cAMP can occur without prolongation of the presynaptic action potential.

\section{Materials and Methods}

Electrophysiological experiments were done on synapses in primary cell culture, generally following the methods of Schacher and Proshansky (1983). Synapses were made between sensory neurons from pleural ganglia and LFS siphon motor neurons from abdominal ganglia of adult Aplysia californica weighing 70-200 gm. Cells for each pair were plated with the cell bodies in contact and synapses formed before any significant amount of process outgrowth had occurred (M. Klein, in preparation). Culture medium was Leibovitz L15 (Sigma) with salts added to give the following final concentrations (in $\mathrm{mm}$ ): $\mathrm{Na}^{+}, 438 ; \mathrm{K}^{+}, 9.5 ; \mathrm{Ca}^{2+}$, $10.5 ; \mathrm{Mg}^{2+}, 52$; HEPES, $9.5 ; \mathrm{pH}$ 7.5. Aplysia hemolymph was added to the culture medium at either $10 \%$ or $50 \%$ in different sets of experiments. Electrophysiological recording was done in the same culture medium, but without hemolymph, using $2 \mathrm{M} \mathrm{KCl}-$ or $\mathrm{K}^{+}$acetate-filled micropipettes connected to Axoclamp-2A amplifiers (Axon Instruments). For voltage clamping, the discontinuous single-electrode mode was used with the sampling frequency set at around $3.5 \mathrm{kHz}$. Postsynaptic neurons were held at $-80 \mathrm{mV}$ in all experiments, and all experiments were done at room temperature. Measurements of amplitudes, areas, and durations were done using the SPIKE electrophysiology analysis program (Hilal Assoc.).

Serotonin (creatinine sulfate; Sigma) and $7 \beta$-desacetyl-7/3-[T-(Nmethylpiperazino)-butyryl] forskolin (7B-forskolin; Calbiochem) were applied to cells in a total volume of $50 \mu \mathrm{l}$ from a hand-held Pipetman. The final concentration around the cells was estimated from independent dilution experiments to be about $50 \%$ of the concentration in the $50 \mu \mathrm{l}$ aliquot. The estimated final concentration of 7B-forskolin was $100 \mu \mathrm{M}$ in all the physiological experiments. In the experiments of Figure 2, native forskolin (Calbiochem) and 1,9-dideoxyforskolin (Sigma) were first dissolved in dimethyl sulfoxide (DMSO), diluted in bathing medium, and applied in a $50 \mu \mathrm{l}$ aliquot to the cells, as above. The final estimated concentration of forskolin and 1,9-dideoxyforskolin was 100 $\mu \mathrm{M}$, and the final estimated DMSO concentration was $0.25 \%$. DMSO alone was added at the same concentration in control experiments.

Radioimmunoassay for cAMP was done using a commercially available kit (Biomedical Technologies, Inc., Stoughton, MA). Pleural sensory neuron clusters were digested as for preparation of cell cultures, dissociated by trituration with a fire-polished Eppendorf pipette tip, and cleaned of glial and neuronal debris. Cells were counted into groups of equal numbers for each assay (from 45 to 100 cells in different assays) and each group was transferred into a glass tube in a volume of $10 \mu \mathrm{l}$ of artificial seawater (composition, in mM: $\mathrm{NaCl}, 460 ; \mathrm{KCl}, 10 ; \mathrm{CaCl}_{2}$, $11 ; \mathrm{MgCl}_{2}, 55 ;$ HEPES, $10 ; \mathrm{pH} 7.5$ ). Test solutions were added to each tube in $10 \mu \mathrm{l}$ to give the desircd final concentration of 5-HT and/or 7Bforskolin with 0.5 or $1 \mathrm{~mm} 3$-isobutyl-1-methylxanthine (Calbiochem) and incubated for $5 \mathrm{~min}$ at room temperature. The tubes were then placed in a boiling water bath for $30 \mathrm{sec}$ to stop all reactions and assayed for cAMP content; the assay was done with the additional acetylation step. In most experiments $20 \mathrm{fmol}$ of cAMP was added to each of the tubes to bring the final cAMP concentration into the range where the assay was most sensitive. All measurements were done in duplicate or triplicate and the median value was used in the calculations. Values are expressed as the increase over basal levels, that is, of cells without any addition of 5-HT or 7B-forskolin, and as a percentage of the increase in response to 5-HT at $10^{-4} \mathrm{M}$, which gave the maximal 5-HT response.

Calculations of changes in synaptic potentials, synaptic currents, and duration of action potentials were done in the following manner: the average of five measurements after treatment was divided by the average of either three or five prior measurements (as noted in the text) to give a single value for each experiment; the average of the values for each group of experiments was then used in the statistical tests (two-tailed $t$ test). Numbers in the text and in the figures are mean \pm SEM.

Potentiation by 7B-forskolin of the cAMP increase caused by 5-HT was calculated as a minimal potentiation, as follows:

$$
\text { Potentiation }_{\min }=\frac{\mathrm{cAMP}_{\mathrm{tog}}-\mathrm{cAMP}_{7 \mathrm{~B} \text { - forsk }}}{\mathrm{cAMP}_{5-\mathrm{HT}}},
$$

where $\mathrm{CAMP}_{\text {tog }}$ is the increase in CAMP with application of 5-HT and $7 \mathrm{~B}$-forskolin together and the other values are the increases to each of the agents alone. This minimal measure of potentiation is actually an underestimate of the true potentiation because some portion of the increase caused by $7 \mathbf{B}$-forskolin alone is due to activation of cyclase molecules coupled to 5-HT receptors, while there should be a correction only for stimulation by 7B-forskolin of cAMP production at other cyclase molecules. If it were the case that all of the cyclase molecules were coupled to 5-HT receptors, then the true potentiation would simply be the ratio of $\mathrm{CAMP}_{\text {tug }}$ to $\mathrm{CAMP} \mathrm{S}_{\mathrm{S} \mathrm{HT}}$; this ratio gives a maximal measure of potentiation.

The discussion of potentiation of 5-HT's effect by 7B-forskolin in the text assumes that both substances incrcase cAMP by activating adenylyl cyclase and that the potentiation is the result of synergism at the cyclase. Although it is conceivable that the effects observed in this study may in addition include effects on cAMP phosphodiesterase, the discussion is in terms of cyclase both because a phosphodiesterase inhibitor was used in the assays and also because synergistic activation of cyclase by forskolin and 5-HT is consistent with demonstrations of such an interaction in many other preparations (Seamon and Daly, 1986).

\section{Results}

\section{Facilitation by 7 B-forskolin occurs preferentially at rested synapses}

The effect of raising cAMP levels on synaptic transmission was investigated with the aid of a water-soluble form of the adenylyl cyclase activator forskolin, 7B-forskolin (see Materials and Methods). The ability of 7B-forskolin to mimic synaptic augmentation was tested at sensory neuron synapses that had been subjected to only minimal stimulation before drug application. In a first set of experiments, a single EPSP was elicited in a postsynaptic cell by firing an action potential in the sensory neuron and 7B-forskolin at an estimated final concentration of $100 \mu \mathrm{M}$ was immediately applied. A second EPSP was elicited $30 \mathrm{sec}$ after the first and stimulation was continued at $30 \mathrm{sec}$ intervals. In control experiments the first EPSP was followed with application of bathing medium or with no treatment at all. Application of 7B-forskolin caused almost a doubling of the EPSP compared to control (Fig. 1): the average EPSP (EPSPS $4-8)$ after 7 B-forskolin application $(n=5)$ was $96 \pm 21 \%$ of the 

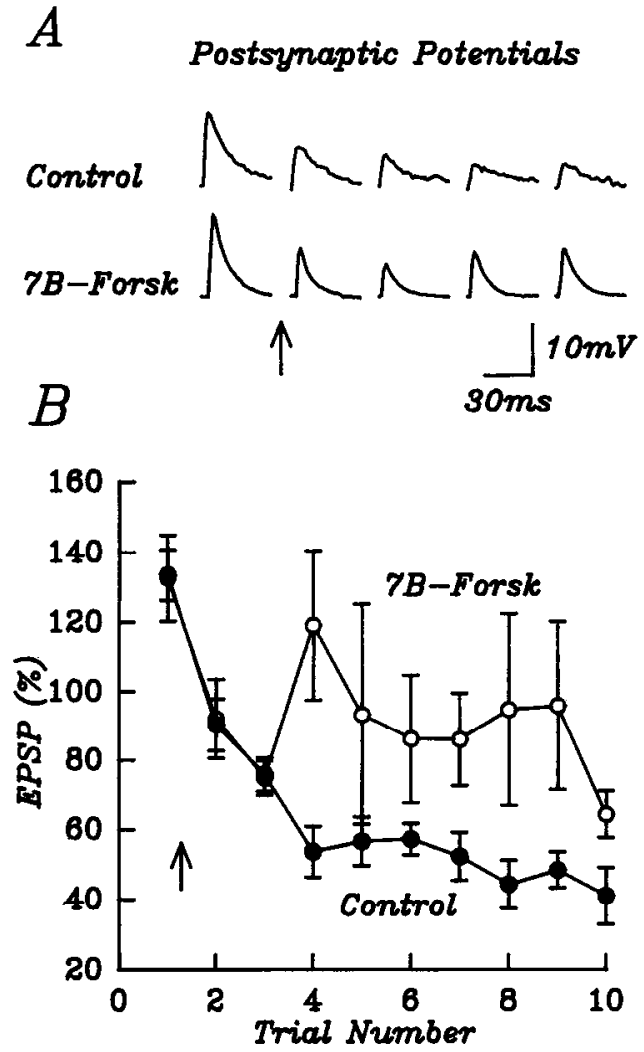

Figure 1. Augmentation by $7 \mathrm{~B}$-forskolin of EPSPs at minimally stimulated synapses. $A$, Single action potentials fired in sensory neurons at $30 \mathrm{sec}$ intervals elicited postsynaptic potentials in LFS motor neurons. Application of 7B-forskolin after the first EPSP in the lower traces led to a reversal of the progressive decline in amplitude characteristic of homosynaptic depression. At the control synapse shown in the upper traces, depression continued monotonically. $B$, Summary data from 5 experimental and 10 control experiments. EPSPs were normalized to the average of the first threc trials.

initial baseline, while the average control $\operatorname{EPSP}(n=10)$ was 53 $\pm 3 \%$ of baseline $(\mathrm{df}=13, t=2.90, p<0.02)$. Synaptic augmentation can thus be mimicked with 7B-forskolin.

To test for specificity of this response to the forskolin analog, this experiment was repeated with 1,9-dideoxyforskolin, an analog that does not activate adenylyl cyclase (Seamon and Daly, 1986). Because the inactive analog is insoluble in aqueous solution, it was necessary to apply it in DMSO. It was therefore also necessary to repeat the experiment using native forskolin in DMSO as well as with DMSO alone.

Facilitation by native forskolin appeared more rapidly after application than was the case with 7B-forskolin (compare Figs. $1,2)$. This difference may perhaps be a consequence of the lipid solubility of native forskolin, a property that would allow it to enter the cell membrane more readily than the water-soluble analog. The average of five EPSPs after forskolin application (EPSPs 3-7) was $143 \pm 29 \%$ of the initial postsynaptic potential, while the corresponding averages after application of the inactive analog and DMSO alone were $58 \pm 9 \%$ and $47 \pm 9 \%$, respectively (Fig. 2). Native forskolin $(n=10)$ caused significant facilitation compared to both the inactive analog $(n=9)$ and to DMSO alone $(n=6)$, while the effects of 1,9-dideoxyforskolin and DMSO alone were not different from each other (forskolin vs. 1,9-dideoxyforskolin: $\mathrm{df}=17, t=2.69, p<0.02$; forskolin

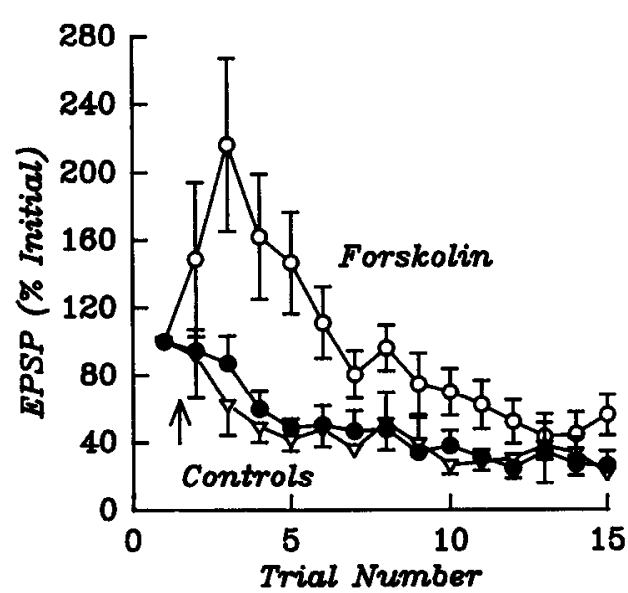

Figure 2. Augmentation by native forskolin, but not by an inactive forskolin analog, of EPSPs at minimally stimulated synapses: summary data from experiments using the same stimulation protocol as in Figure 1 where either native forskolin $(n=10)$, the inactive analog 1,9-dideoxyforskolin ( $n=9$ for trials $1-10$ and $n=8$ for trials 11-15), or DMSO alone $(n=6)$ was applied after the first EPSP. Native forskolin (open circles) and the analog (solid circles) were applied at an estimated final concentration of $100 \mu \mathrm{M}$ in $0.25 \%$ DMSO, and DMSO alone (triangles) was applied at an estimated final concentration of $0.25 \%$. EPSPs were normalized to the first EPSP of cach experiment.

vs. DMSO: $\mathrm{df}=14, t=2.51, p<0.05 ; 1$,9-dideoxyforskolin vs. DMSO: $\mathrm{df}=13, t=0.84, p>0.25$ ).

In contrast to the results at minimally stimulated synapses, 7B-forskolin had no effect on transmission at synapses that had been activated repeatedly (Fig. 3). Fifteen action potentials were fired in the sensory neuron at $30 \mathrm{sec}$ intervals, causing synaptic depression to about $30 \%$ of the amplitude of the first EPSP of the experiment (not shown); at that time, 7B-forskolin was applied and the stimulation continued as previously $(n=6)$. In control experiments using other synapses $(n=7)$ an identical volume of bathing medium was applied instead of 7B-forskolin. The avcrage of five EPSPs (EPSPs 18-22) after application of $7 \mathrm{~B}$-forskolin was $86 \pm 7 \%$ of the average of the five EPSPs immediately preceding the application, while in the control experiments the corresponding value was $90 \pm 9 \%(\mathrm{df}=11, t=$ $0.19, p>0.5$ ). 5-HT was applied at the end of every experiment to ensure that the synapse could undergo facilitation by $5-\mathrm{HT}$. Thus, 7B-forskolin does not mimic 5-HT in inducing synaptic restoration.

There are two sets of reasons for suspecting that the synaptic augmentation caused by $7 \mathrm{~B}$-forskolin at relatively rested synapses (Fig. 1) might have been underestimated. Because 7Bforskolin does not mimic synaptic restoration, it is possible that the repeated activation of the synapse at $30 \mathrm{sec}$ intervals might attenuate its effect. In addition, because the initial EPSPs in an experiment are generally larger than later ones, enhancement by $7 \mathrm{~B}$-forskolin might be reduced as a result of membrane rectification or of approach to the reversal potential. Both of these concerns were addressed in the following set of experiments. The postsynaptic cell was voltage clamped at a potential hyperpolarized from rest and the postsynaptic current response (EPSC) to a single presynaptic action potential was monitored before 7B-forskolin was applied. A single additional response was then elicited, at an interval of $2 \mathrm{~min}$ after the first. In control experiments without $7 \mathrm{~B}$-forskolin the total net charge transferred (EPSC area) in the second response was $90 \pm 18 \%$ that 
$A$

Postsymaptic Potentials

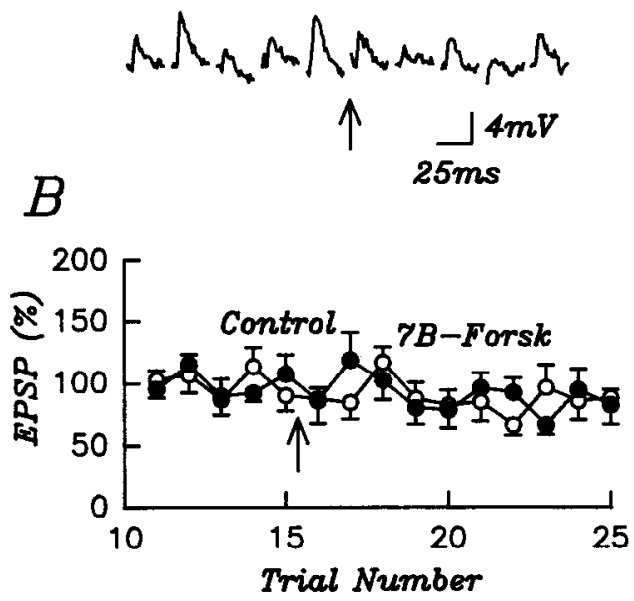

Figure 3. Failure of 7B-forskolin to restore EPSPs at more extensively activated synapses. $A$, Fifteen EPSPs were elicited at $30 \mathrm{sec}$ intervals before application of $100 \mu \mathrm{M}$ 7B-forskolin at the arrow. Shown are EPSPs 11-20. The large variability in amplitude is characteristic of smaller EPSPs at these synapses. $B$, The graph shows the average of EPSPs 1125 in seven experiments with application of 7B-forskolin (open circles) and the average of six control experiments. At the arrow either $100 \mu \mathrm{M}$ 7B-forskolin or an identical volume of bathing medium was applied. The average of EPSPs 11-15 was taken as $100 \%$.

of the first $(n=8)$. When 7B-forskolin was applied between the first and the second stimuli, however $(n=7)$, the second response of the pair was $154 \pm 19 \%$ of the first (Fig. 4 ; df $=13$, $t=2.45, p<0.05)$. There was no significant change in presynaptic membrane potential between the first and the second trial [the mean depolarization was $0.94 \pm 0.77 \mathrm{mV}$ for the treated $(n=7)$ and $0.27 \pm 0.44 \mathrm{mV}$ for the controls $(n=7) ; \mathrm{df}=12$, $t=0.76, p>0.25]$. Measuring the peak EPSC rather than the total charge yielded a comparable difference between the 7Bforskolin-treated preparations and the controls (Fig. 4; $82 \pm$ $15 \%$ for controls vs. $172 \pm 18 \%$ for experimentals; $\mathrm{df}=13, t$ $=3.97, p<0.005$ ). Enhancement of the synaptic response by 7B-forskolin was thus about the same as in the first set of experiments where the synaptic potential rather than the EPSC was measured.

\section{Synaptic augmentation by 7 B-forskolin can occur without an increase in duration of action potentials}

Examination of the action potentials in these experiments yielded additional information that addressed two separate questions concerning the mechanism of action of 7B-forskolin. In several preparations, the native, water-insoluble form of forskolin has been reported to depress potassium currents directly, independent of the increase in cAMP that it causes (Laurenza et al., 1989; Baxter and Byrne, 1990). One concern in the present cxpcriments, therefore, was that this water-soluble form as well might directly depress potassium currents and thereby broaden action potentials and enhance synaptic release in a cAMP-independent manner. That this is not the mechanism of action of 7B-forskolin in these experiments is indicated by two sets of observations. First, in the experiments where augmentation of the synaptic potential was examined, the enhancement of the EPSP was not correlated with the spike broadening caused by $7 \mathrm{~B}$-forskolin. Thus, in the experiments shown in Figure $1 B$,

\section{Postsynaptic Current}
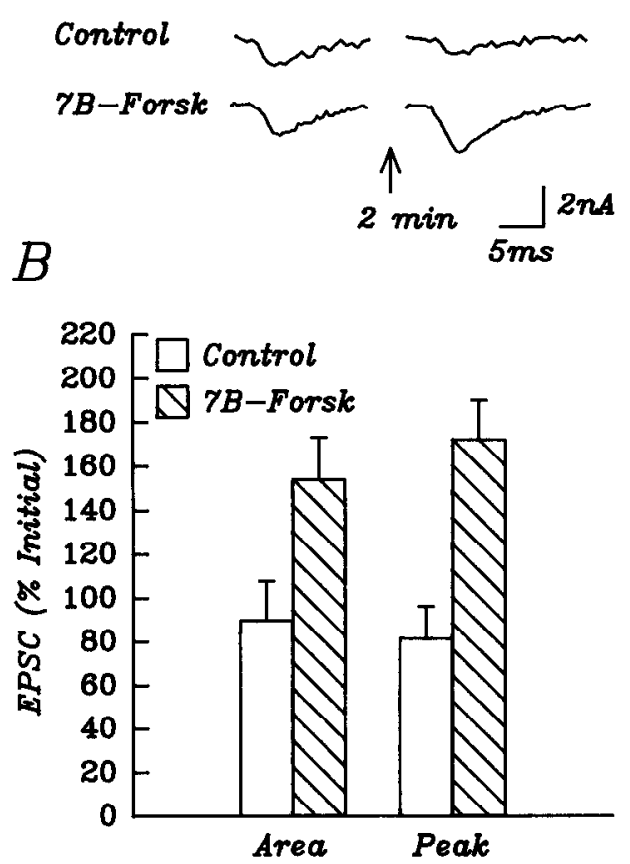

Figure 4. Augmentation of EPSCs by 7B-forskolin at rested synapses. $A$, Records from one control experiment and one application of $7 \mathrm{~B}$ forskolin in a different experiment. A single EPSC was elicited and either 7B-forskolin or bathing medium was immediately applied. A second EPSC was elicited at $2 \mathrm{~min}$ after the first. The EPSC declined in the control while it increased after application of 7B-forskolin. $B$, Summary data showing augmentation of EPSCs by 7B-forskolin in seven experiments and decline of EPSCs in eight control experiments. Both the peak of the second EPSC of each pair and the total charge transferred (EPSC area) are expressed as a percentage of the initial EPSC value.

despite the fact that the first and the second action potentials after 7B-forskolin application showed average broadening of $12 \%$ and $16 \%$, respectively, as compared to the initial spike (not shown), the corresponding average synaptic potentials are identical to the controls, where the broadening was $1 \%$ for both. More striking, however, is the fact that there was no difference between the third action potential after $7 \mathrm{~B}$-forskolin application and the second (the ratio of spike widths was $1.005 \pm 0.007$ ), whereas the EPSP evoked by the third action potential was now double the control.

Furthermore, in the experiments where the interstimulus interval was $2 \mathrm{~min}$ and the EPSC increased by over $50 \%$ after $7 \mathrm{~B}$ forskolin application, there was no significant broadening of the second action potential with respect to the first (Fig. 5). In fact, when the action potential duration was measured at three different points-at $30 \%, 50 \%$, and $70 \%$ of peak amplitude-the average duration after $7 \mathrm{~B}$-forskolin application was actually slightly less at the two more depolarized levels (the difference was not statistically significant).

\section{Synergistic activation of adenylyl cyclase by 5-HT and $7 B$-forskolin}

The finding that $7 \mathrm{~B}$-forskolin can increase transmission at rested, but not at extensively activated, synapses suggests that synaptic restoration may not be mediated through cAMP. However, since other interpretations of this differential effect of $7 \mathrm{~B}$-for- 


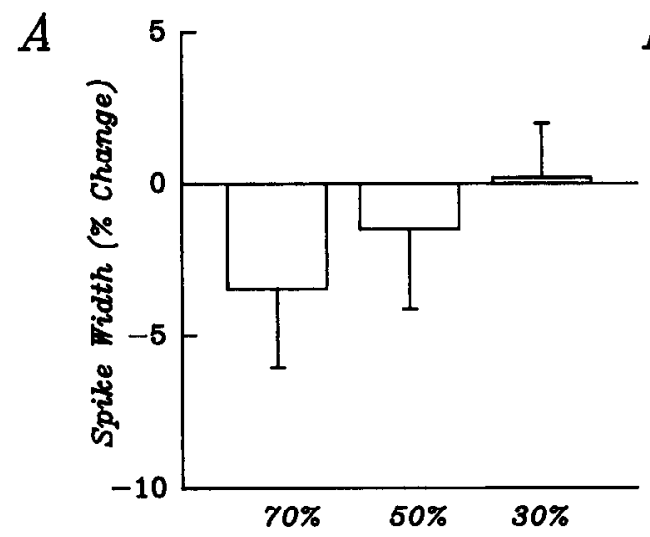

$B$

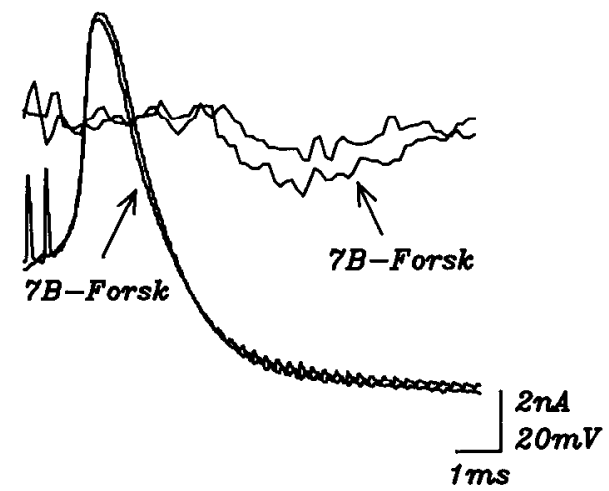

Figure 5. Augmentation of EPSCs by $7 \mathrm{~B}$-forskolin is not accompanied by prolongation of action potentials. $A$, Action potential duration in the sensory neurons of the experiments of Figure 3 was measured as the time elapsed from the peak of the spike to different percentages of the peak amplitude during the falling phase. Despite the augmentation of the EPSC caused by 7B-forskolin, the second of the pair of action potentials showed a slight narrowing compared to the initial action potential at the two more depolarized levels $(70 \%$ and $50 \%$ of peak amplitude) and practically no change at $30 \%$. $B$, Action potentials and EPSCs recorded from a synapse where augmentation of the EPSC by 7B-forskolin was accompanied by narrowing of the action potential. skolin are possible, it was necessary to examine the changes in cAMP level caused by 7B-forskolin and by 5-HT. Radioimmunoassay for cAMP was therefore performed on groups of dissociated sensory neurons prepared in the same way as those uscd for making cultured synapses. These neurons were separated from the glial cells, sheath components, dead cells, and obvious nonsensory neurons that generally contaminate the sensory neuron clusters used for biochemical assays to ensure that the changes in cAMP levels induced by 7B-forskolin and 5-HT in the present experiments were accurate indications of the concentration in the sensory neurons only. In addition, treating the cells for the biochemical assays in the same way as those used in the electrophysiological experiments decreased the likelihood that the cells in the two types of experiment might respond differently. In some cases, cells used for electrophysiological experiments were taken from the same population of sensory neurons as those used in the radioimmunoassay.
5-HT and 7B-forskolin generally caused comparable increases in the total amount of CAMP (Fig. 6). However, since 7B-forskolin presumably also acts at cyclase molecules besides those coupled to 5-HT receptors, comparable increases in total cAMP might not reflect the local concentrations in different parts of the cell. In particular, it is possible that some of the sites of 5-HT sensitivity might be clustered near sites of synaptic contact, thus giving rise to a much higher concentration of cAMP locally than is achieved by a treatment with 7B-forskolin that gives the same overall increase. A mismatch between the degrees of synaptic facilitation induced by 7B-forskolin and by 5-HT would then not necessarily have any bearing on the question of the involvement of cAMP in facilitation. This argument would not apply as strictly to facilitation caused by action potential broadening, since modulation of potassium channels would not have to occur right at the synapse to prolong the action potential at the release sites. The problem of the spatial distribution of
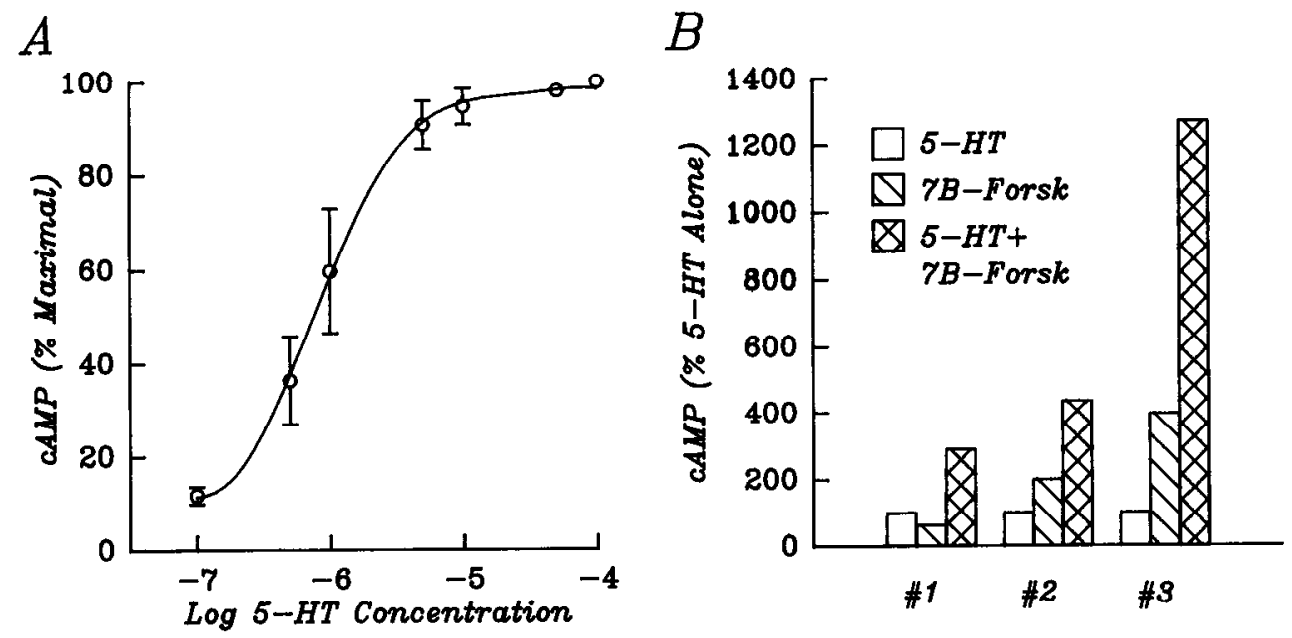

Figure 6. Stimulation of cAMP by 5-HT and by 7B-forskolin, and synergistic stimulation by 5-HT and 7B-forskolin in combination. $A$, Relationship between increase in cAMP content and 5-HT concentration in sensory neurons. The points are averages from three separate experiments; cAMP is expressed as increases over untreated controls normalized to the increase caused by $10^{-4} \mathrm{M} 5-\mathrm{HT}$. Half-maximal stimulation was achieved at about $0.8 \mu \mathrm{M}$ 5-HT. The error bars (showing SEM) for the point at $5 \times 10^{-5} \mathrm{M}$ fall within the symbol and do not appear. $B$, Synergistic stimulation of cAMP by combined application of 5-HT and 7B-forskolin. Each set of bars represents the average of two experiments where cAMP levels in response to 5-HT alone, 7B-forskolin alone, and 5-HT and 7B-forskolin together were measured. The cAMP increase in response to 5-HT alone in each experiment is taken as $100 \%$. In all cases, combined application resulted in stimulation that was greater than the sum of the stimulations caused by separate applications. In experiment \#1,5-HT concentration was $2 \mu \mathrm{M}$ and $7 \mathrm{~B}$-forskolin was $20 \mu \mathrm{M}$; in experiments \#2 and \#3 5-HT was $1 \mu \mathrm{M}$ and $7 \mathrm{~B}$-forskolin was $100 \mu \mathrm{M}$ and $200 \mu \mathrm{M}$, respectively. 
Figure 7. Synaptic restoration by 5-HT alone and by 5 -HT applied together with 7B-forskolin: absence of synergism. $A$ Fifteen F.PSPs were elicited at $30 \mathrm{sec}$ intervals, at which time (arrow) either 5-HT alone ( $1 \mu \mathrm{M}$ estimated final concentration) or $5-\mathrm{HT}$ plus $7 \mathrm{~B}$-forskolin (100 $\mu \mathrm{M}$ estimated final concentration) was applied. The graph shows summary data for EPSPs 11-20 for seven experiments with 5-HT alone and eight experiments with combined application. The average facilitation in the two sets of experiments is virtually identical. As before, the average of EPSPs $11-$ 15 was taken as $100 \%$. B. Action potential duration at $30 \%$ of peak amplitude (measured as in Fig. 5) in the same experiments as $A$. Broadening by 5-HT was greatly potentiated when $7 \mathrm{~B}$-forskolin was also present.

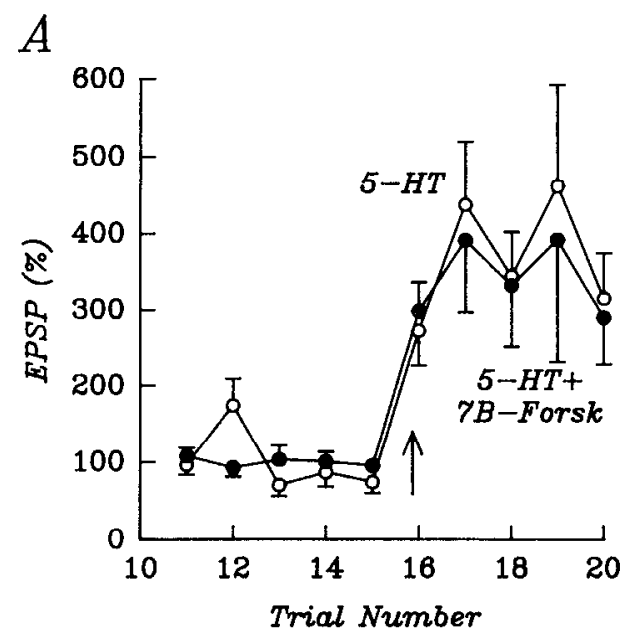

cAMP thus arises primarily when facilitation is largely independent of prolongation of the action potential-as has been shown to be the case for synaptic restoration. (The results discussed above in relation to synaptic augmentation argue that even augmentation is likely to be at least partially mediated by processes independent of spike broadening.) The different effectiveness of $7 \mathrm{~B}$-forskolin under the two different conditions could also result from, for example, a greater affinity for protein kinase A phosphorylation of the substrates that are involved in augmentation (Goldsmith and Abrams, 1992), or perhaps by a difference in the amount of cAMP generated at relatively rested synapses as compared to synapses that had been stimulated more.

In many systems, activation of adenylyl cyclase by ligands is potentiated by forskolin, indicating that the two agents are acting at the same cyclase molecule (Seamon and Daly, 1986). Thus, synergistic activation of eyclase in the sensory neurons by $7 \mathrm{~B}-$

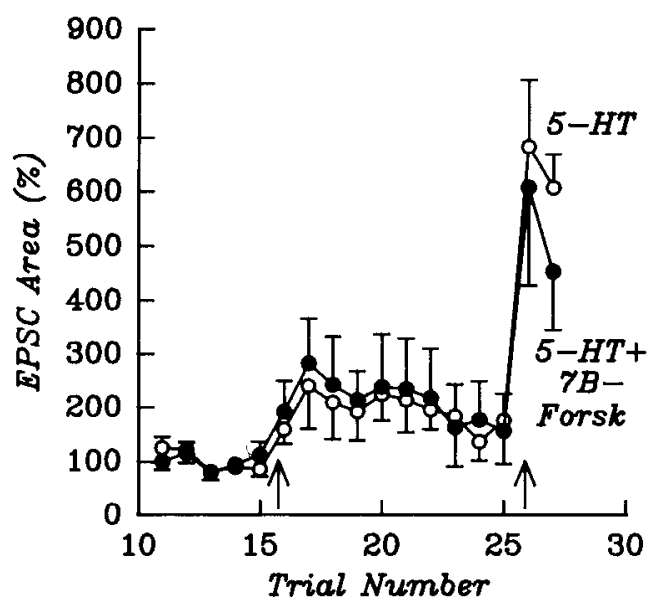

Figure 8. Absence of synergism in synaptic restoration by a lower concentration of 5-HT applicd together with $7 \mathrm{~B}$-forskolin. The estimated final concentration of 5-HT in these experiments was $0.5 \mu \mathrm{M}$, and of $7 \mathrm{~B}$-forskolin, $100 \mu \mathrm{M}$. As before, there was no difference between facilitation caused by 5 -HT alone $(n=9)$ and in combination with 7Bforskolin $(n=8)$ when applied after 15 stimuli at $30 \mathrm{sec}$ intervals. At the second arrow a maximally effective concentration of 5-HT $(10 \mu \mathrm{M})$ caused additional facilitation, indicating that the facilitation caused by the lower concentration was not at saturation. forskolin and 5-HT applied together would indicate action at the same site, and the problem of differences in the spatial distribution of cAMP would thus be circumvented. To make a more meaningful comparison between the results of cAMP assays and the degree of facilitation of the synaptic responses, increases in cAMP caused by combined application of $7 \mathrm{~B}$-forskolin and 5-HT were therefore measured. 5-HT and 7B-forskolin do in fact act synergistically in increasing cAMP in the sensory neurons (Fig. 6B). In a number of assays comparing the rises in CAMP caused by $7 \mathrm{~B}$-forskolin and 5 -HT alone and in combination, the minimal potentiation of the response to $5-\mathrm{HT}$ caused by concomitant application of $7 \mathrm{~B}$-forskolin varicd from greater than 2-fold to greater than 10-fold (sce Materials and Methods for calculation). Examining the effects on synaptic transmission of combined versus separate application should therefore give some indication of the involvement of cAMP in the facilitation related to synaptic restoration.

\section{Synaptic restoration by $5-H T$ is not potentiated by 7 B-forskolin}

In the next series of experiments, synapses were activated as previously by eliciting 15 EPSPs with a $30 \mathrm{sec}$ interstimulus interval. At this time, either 5-HT alone or 5-HT in combination with $7 \mathrm{~B}$-forskolin was applied, and the facilitation under the two conditions compared. As is evident in Figure $7 . A, 5-H T$ alone at an estimated final concentration of $1 \mu \mathrm{M}$ caused the same enhancement in the EPSP as did joint application of 5-HT and $100 \mu \mathrm{M} 7 \mathrm{~B}$-forskolin.

In these experiments, action potential broadening by $5-\mathrm{HT}$ alone and by 5 -HT plus 7 B-forskolin was examined to determine whether there was any effect of 7B-forskolin on 5-HTinduced broadening. Action potential broadening at $30 \%$ of spike amplitude (action potentials 18-20) was $5 \pm 1 \%$ with 5 -HT ( $n$ $=7)$ and $28 \pm 3 \%$ with 5 -HT in combination with $7 \mathrm{~B}$-forskolin $(n=8 ; \mathrm{d} \Gamma=13, l=6.86, p<0.001)$ (Fig. $7 B)$. In the experiments of Figure 3 where $7 \mathrm{~B}$-forskolin alone was tested after 15 stimuli, action potential broadening was approximately $8 \%$ (not shown). Thus, there was a synergistic action of $7 \mathrm{~B}$-forskolin with 5 -HT on the action potential, whereas there was no interaction at all on synaptic transmission in the same experiments. Although it is not a necessary conclusion that the interaction on the action potential is mediated by cAMP, this synergistic interaction is consistent with the biochemical synergism, and suggests that the 
cAMP level is greatly enhanced on exposure of the neurons to the combination of 5-HT and 7B-forskolin. In addition, the failure of a greatly enhanced broadening of the action potential to result in a larger facilitation implies that the contribution of broadening to synaptic restoration under the conditions of these experiments is not a major one.

To ensure that the failure of 7B-forskolin to amplify the facilitation induced by 5-HT was not a consequence of saturation of the facilitatory response, a second set of experiments was done using 5-HT at an estimated final concentration of $0.5 \mu \mathrm{M}$. In this set of experiments also, facilitation induced by joint application of 5-HT and 7B-forskolin was no greater than that induced by 5 -HT alone (Fig. 8). As a final check, a maximally effective dose of $5-\mathrm{HT}, 10 \mu \mathrm{M}$, was added at the end of each experiment to show that facilitation was far from saturation (Fig. 8, second arrow).

\section{Discussion}

Taken together, the CAMP assays showing a powerful synergism between 7B-forskolin and 5-HT and the absence of any interaction between 5-HT and 7B-forskolin in the synaptic restoration response make it difficult to argue that an increase in cAMP is responsible to an important degree for the facilitation caused by 5-HT at synapses that have undergone repeated activation. The ability of $7 \mathrm{~B}$-forskolin to mimic synaptic augmentation at relatively rested synapses, in contrast, is consistent with a role for cAMP in these conditions. One would expect, moreover, that after intermediate amounts of synaptic activation, the contribution of cAMP to facilitation would lie between these extremes.

The failure of a forskolin analog that does not activate adenylyl cyclase to mimic synaptic augmentation (Fig. 2) suggests that the effects of 7B-forskolin and native forskolin are the result of an increase in cAMP. Since intracellular injection of cAMP into the sensory neurons (Brunelli et al., 1976) causes synaptic facilitation, the present results are consistent with a presynaptic site of action for the active forms of forskolin. However, because the activators of adenylyl cyclase were applied extracellularly here, the present results do not exclude the possibility that there may also be a postsynaptic site of action of these agents in the enhancement of synaptic transmission at relatively rested synapses. If there is in fact a postsynaptic effect of activating the cyclase, then the results reported here argue that this effect must also be sensitive to the amount of prior stimulation because $7 \mathrm{~B}$-forskolin is without effect at synapses that have been stimulated repeatedly (Figs. 3, 7, 8). In any case, whether or not a postsynaptic site is involved in augmentation by $7 \mathrm{~B}$-forskolin, the conclusion that an increase in cAMP is not sufficient to cause synaptic restoration is not affected by this possibility.

The data presented here are consistent with earlier work showing that facilitation by $5-\mathrm{HT}$ has different mechanisms at rested and at depressed synapses (Hochner et al., 1986b; Braha et al., 1990). However, the present findings suggest that the relevant variable determining which of the facilitatory processes predominates may not be the amount of depression per se. In the summary graphs shown in Figures 1 and 2, where 7B-forskolin or native forskolin was added after only a single EPSP had been elicited, synaptic augmentation is apparent even when the control EPSP has been depressed to less than $35 \%$ of the initial EPSP. In Figure 3, by contrast, where the EPSP had been depressed to $29 \%$ of the initial value, the effect of $7 \mathrm{~B}$-forskolin is completely blocked. The assay of Braha et al. (1990) shows little difference between the behavior of synapses that have been depressed to these two levels. The differential effect of 7B-forskolin here suggests, therefore, that it may be the amount of prior activation, rather than the extent of depression, that determines whether cAMP-dependent facilitation can take place. Since, in general, the degree of synaptic depression is correlated with the amount of prior activation, it might appear that the effectiveness of cAMP depends on the degree of depression. Another possible explanation is that cAMP might be effective in preventing synaptic depression even though it is unable to reverse depression once it has occurred. The data of Figure $1 B$ appear to show some degree of reversal of synaptic depression between the third and the fourth EPSP, suggesting that this latter explanation cannot be correct in an absolute sense, but it is still possible that cAMP is much less effective in reversing depression than it is in preventing its progression. Finally, as a corollary to these two possibilities, synaptic depression may itself comprise more than one process, only one of which may be susceptible to reversal by cAMP.

The finding that facilitation is likely to have at least two different biochemical mechanisms is consistent with earlier reports indicating roles for both protein kinase $A$ and protein kinase C (Braha et al., 1990; Sacktor and Schwartz, 1990; Sugita et al., 1992). Bailey and Chen (1988) showed that short-term synaptic depression is accompanied by depletion of synaptic vesicles from the regions immediately adjacent to the presumed presynaptic release sites. If this local depletion is the only determinant of synaptic depression, then the differential effects of activating adenylyl cyclase reported here suggest that CAMP may not be capable of rapid replenishment of an immediately releasable pool of neurotransmitter, but that it must act at some other step in the release process to enhance release. On the other hand, the finding that activation of protein kinase $C$ can mimic synaptic restoration (Braha et al., 1990) argues that the local vesicle depletion accompanying short-term synaptic depression can be reversed by activation of this kinase system.

In contrast to the present results, a number of other studies conclude that cAMP does play a role in synaptic restoration (Castellucci et al., 1980, 1982; Braha et al., 1990; Schacher et al., 1990; Goldsmith and Abrams, 1991). These experiments fall into two classes: one set uses artificial increases in cAMP to mimic restoration, and the other makes use of blockers of different steps in the cAMP cascade to block synaptic restoration. A number of possible explanations for these discrepancies can be suggested. One likely possibility, based on the discussion above, is that the discrepancies arise from the fact that the different experiments were done after different amounts of synaptic activation and using different stimulus frequencies. Thus, at synapses that had been activated less than those in the present study, cAMP could still be evoking the process of synaptic augmentation despite the fact that the degree of synaptic depression might be similar.

A different kind of explanation might also account for the results of some of the blocking experiments in which it was found that blockade either of cAMP-dependent protein kinase or of adenylyl cyclase blocked facilitation even at repeatedly stimulated synapses: it is possible that an increase in cAMP, while not being sufficient to restore transmission at depressed synapses by itself, might nonetheless be necessary for synaptic restoration to be effective. Another more trivial possibility is that the blockers may act at sites besides those involved in the cAMP pathway - at other kinases, for example. 
An alternate interpretation of the difference between the effects of $7 \mathrm{~B}$-forskolin at relatively rested and at more extensively stimulated synapses is that the increase in cAMP is smaller after morc stimulation. Although this possibility has not been tested directly, the fact that 7B-forskolin causes action potential prolongation after 15 stimuli as well as at more rested synapses (with a $30 \mathrm{sec}$ interstimulus interval) argues against it.

A second implication of these findings is that facilitation by cAMP may not depend on action potential broadening alone, but may have some other mechanism of action. Figure $5 \mathrm{~A}$ shows the changes in action potential duration caused by $7 \mathrm{~B}$-forskolin at three different points: there are slight decreases in duration at $70 \%$ and $50 \%$ of peak amplitude, and no change at $30 \%$.

The three points at which action potential duration was measured correspond roughly to membrane potentials of $-15,+5$ and $+25 \mathrm{mV}$. Since the calcium current that is responsible for transmitter release begins to activate significantly at about -10 $\mathrm{mV}$ and peaks between approximately +10 and $+20 \mathrm{mV}$ (Edmonds et al., 1990), it would be difficult to maintain that prolongation of the calcium current as a rcsult of spike broadening is responsible for the increase in synaptic transmission in these experiments. This conclusion is particularly compelling because, in these experiments, the gross morphology of the neuron pairs suggests that the action potential recorded by the microelectrode is the same as that occurring at the site of transmitter release (see Materials and Methods). The broadening observed when the interstimulus interval was $30 \mathrm{sec}$ is likely to result from the combination of the action of $7 \mathrm{~B}$-forskolin with a progressive voltage-dependent inactivation of potassium currents, whereas the triggering of only two action potentials with a 2 min interstimulus interval in the other experiments minimized such inactivation. It is also possible that the greater accumulation of calcium resulting from the higher frequency of stimulation potentiates the activation of the cyclase by $7 \mathrm{~B}$-forskolin, as has been proposed to occur with 5-HT stimulation of the cyclase (Abrams et al., 1991).

In the experiments of Figure $1 B$ an opposite relationship between action potential broadening and synaptic augmentation is seen: after 7B-forskolin application spike duration increased by $12 \%$ and $16 \%$ in trials 2 and 3 , respectively (not shown), without any increase in the EPSP as compared to the controls. The lack of any obvious relationship between action potential prolongation and augmentation of the synaptic response in the two sets of experiments suggests that the contribution of action potential broadening to synaptic augmentation warrants further examination. Though it appears that action potential broadening is not necessary for synaptic augmentation, the possibility that there may still be some additional contribution of broadening to this form of facilitation cannot conclusively be rejected.

The contribution of action potential prolongation to synaptic restoration is also called into question by the results of the present study: as is shown in Figure 7, 7B-forskolin can apparently potentiate 5-HT's ability to prolong action potentials without thereby increasing synaptic restoration.

A dissociation between spike broadening and synaptic facilitation has also been reported by Sugita et al. (1992). They showed that staurosporine, which they used as an inhibitor of protein kinase $\mathrm{C}$, can block prolongation by 5-HT of sensory neuron action potentials without blocking synaptic facilitation in intact Aplysia ganglia. If staurosporine blocks spike broadening in the synaptic terminals as well as in the cell bodies, then their results imply that facilitation by $5-\mathrm{HT}$ does not require broadening even at relatively rested synapses. If, in addition, staurosporine is specific in its inhibition of protein kinase $C$, their results are also consistent with the conclusion of the present report that cAMP can cause facilitation through a mechanism independent of spike broadening.

The results of the present study imply that of the facilitatory processes triggered by $5-\mathrm{HT}$, only the one involved in augmentation of synaptic transmission at relatively rested synapses may be caused by an increase in cAMP concentration in the sensory neurons. In contrast, the facilitatory process (or processes) involved in restoration of transmission at synapses that have undergone more extensive activation depends on factors other than cAMP. The behavioral implication of these findings is that a transmitter-mediated increase in cAMP in the sensory neurons may contribute to sensitization of a reflex that has not been habituated, whereas dishabituation of a habituated response does not depend on an increase in cAMP in these neurons except, possibly, as a necessary cofactor that does not control the magnitude of the reflex enhancement. In addition, the results suggest that synaptic augmentation by cAMP can occur without action potential prolongation, and that there may be little contribution of action potential broadening to synaptic restoration.

The results of this study, in combination with earlier work (Hochner et al., 1986b; Braha et al., 1990; Sacktor and Schwartz, 1990; Sugita et al., 1992), suggest that facilitation by 5-HT may be analyzable into at least three components: a process independent of cAMP - perhaps mediated by protein kinase $\mathrm{C}$ - that is responsible for synaptic restoration at extensively stimulated synapses, and two cAMP-dependent processes that contribute to synaptic augmentation, one that may act through action potential prolongation and the other that is independent of it. Thus, not only can more than one biochemical mechanism contribute to synaptic facilitation and to behavioral plasticity in Aplysia, but activation of even one of the biochemical pathways may induce changes at a number of the physiological loci involved in learning.

\section{References}

Abrams TW, Karl KA, Kandel ER (1991) Biochemical studies of stimulus convergence during classical conditioning in Aplysia: dual regulation of adenylate cyclase by $\mathrm{Ca}^{2+} /$ calmodulin and transmitter. J Neurosci 11:2655-2665.

Bailey CH, Chen M (1988) Morphological basis of short-term habituation in Aplysia. J Neurosci 8:2452-2459.

Baxter DA, Byrne JH (1990) Reduction of voltage-activated $\mathrm{K}^{+}$currents by forskolin is not mediated via cAMP in pleural sensory neurons of Aplysia. J Neurophysiol 64:1474-1483.

Bernier L, Castellucci VF, Kandel ER, Schwartz JH (1982) Facilitatory transmitter causes a selective and prolonged increase in adenosine 3:5-monophosphate in sensory neurons mediating the gill- and siphon-withdrawal reflex in Aplysia. J Neurosci 2:1682-1691.

Braha O, Dale N, Hochner B, Klein M, Abrams TW, Kandel ER (1990) Second messengers involved in the two processes of presynaptic facilitation that contribute to sensitization and dishabituation in Aplysia sensory neurons. Proc Natl Acad Sci USA 87:2040-2044.

Brunelli M, Castellucci V, Kandel ER (1976) Synaptic facilitation and behavioral sensitization in Aplysia: possible role of serotonin and cyclic AMP. Science 194:1178-1181.

Castellucci VF, Kandel ER (1974) A quantal analysis of the synaptic depression underlying habituation of the gill-withdrawal reflex in Aplysia. Proc Natl Acad Sci USA 71:5004-5008.

Castcllucci VF, Kandel ER (1976) Presynaptic facilitation as a mechanism for behavioral sensitization in Aplysia. Science 194:1176-1178.

Castellucci VF, Kandel ER, Schwartz JH, Wilson FD, Nairn AC, Greengard $P$ (1980) Intracellular injection of the catalytic subunit of cyclic AMP-dependent protein kinase stimulates facilitation of transmitter release underlying behavioral sensitization in Aplysia. Proc Natl Acad Sci USA 77:7492-7496.

Castellucci VF, Nairn AC, Greengard P, Schwartz JH, Kandel ER (1982) Inhibitor of adenosine $3^{\prime}: 5$-monophosphate-dependent protein ki- 
nase blocks presynaptic facilitation in Aplysia. J Neurosci 2:16731681 .

Dixon D, Atwood HL (1989a) Adenylate cyclase system is essential for long-term facilitation at the crayfish neuromuscular junction. $J$ Neurosci 9:4246-4252.

Dixon D, Atwood HL (1989b) Phosphatidylinositol system's role in serotonin-induced facilitation at the crayfish neuromuscular junction. J Neurophysiol 62:239-246.

Dixon D, Atwood HL (1989c) Conjoint action of phosphatidylinositol and adenylate cyclase systems in serotonin-induced facilitation at the crayfish neuromuscular junction. J Neurophysiol 62:1251-1259.

Edmonds B, Klein M, Dale N, Kandel ER (1990) Contributions of two types of calcium channels to synaptic transmission and plasticity. Science 250:1142-1147.

Etchcbcrrigaray R, Matzel LD, Lederhendler II, Alkon DL (1992) Classical conditioning and protein kinase $C$ activation regulate the same single potassium channel in Hermissenda crassicornis photoreceptors. Proc Natl Acad Sci USA 89:7184-7188.

Gingrich KJ, Byme JH (1985) Simulation of synaptic depression, posttetanic potentiation and presynaptic facilitation of synaptic potentials from sensory neurons mediating gill-withdrawal reflex in Aplysia. J Neurophysiol 53:652-669.

Glanzman DL, Mackey SL, Hawkins RD, Dyke AM, Lloyd PE, Kandel ER (1989) Depletion of serotonin in the nervous system of Aplysia reduces the behavioral enhancement of gill withdrawal as well as the heterosynaptic facilitation produced by tail shock. J Neurosci 9:42004213.

Goldsmith BA, Abrams TW (1991) Reversal of synaptic depression by serotonin at Aplysia sensory neuron synapses involves activation of adenylyl cyclase. Proc Natl Acad Sci USA 88:9021-9025.

Goldsmith BA, Abrams TW (1992) Cyclic AMP modulates multiple $\mathrm{K}^{+}$currents, increasing spike duration and excitability in Aplysia sensory neurons. Proc Natl Acad Sci USA 89:11481-11485.

Hawkins RD, Abrams TW, Carew TJ, Kandel ER (1983) A cellular mechanism of classical conditioning in Aplysia: activity-dependent amplification of presynaptic facilitation. Science 219:400-405.

Hochner B, Klein M, Schacher S, Kandel ER (1986a) Action potential duration and the modulation of transmitter release from the sensory neurons of Aplysia in presynaptic facilitation and behavioral sensitization. Proc Natl Acad Sci USA 83:8410-8414.

Hochner B, Klein M, Schacher S, Kandel ER (1986b) Additional component in the cellular mechanism of presynaptic facilitation contributes to behavioral dishabituation in Aplysia. Proc Natl Acad Sci USA 83:8794-8798.

Klein M, Kandel ER (1980) Mechanism of calcium current modulation underlying presynaptic facilitation and behavioral sensitization in Aplysia. Proc Natl Acad Sci USA 77:6912-6916.

Klein M, Shapiro E, Kandel ER (1980) Synaptic plasticity and the modulation of the $\mathrm{Ca}^{2+}$ current. J Exp Biol 89:117-157.

Laurenza A, Sutkowski EM, Seamon KB (1989) Forskolin: a specific stimulator of adenylyl cyclase or a diterpene with multiple sites of action? Trends Pharmacol Sci 10:442-447.
Malenka RC, Kauer JA, Perkel DJ, Mauk MD, Kelly PT, Nicoll RA (1989) An essential role for postsynaptic calmodulin and protein kinase activity in long-term potentiation. Nature 340:554-557.

Malinow R, Schulman H, Tsien RW (1989) Inhibition of postsynaptic PKC or CaMKII blocks induction but not expression of LTP. Science 245:862-866.

Matzel LD, Lederhendler II, Alkon DL (1990) Regulation of shortterm associative memory by calcium-dependent protein kinase. $\mathrm{J}$ Neurosci 10:2300-2307.

Ocorr KA, Byrne JH (1985) Membrane responses and changes in cAMP levels in Aplysia sensory neurons by serotonin, tryptamine FMRFamide and small cardinactive peptide $\left(\mathbf{S C P}_{\mathrm{B}}\right)$. Neurosci Lett 55:113-118.

O'Dell TJ, Hawkins RD, Kandel ER, Arancio O (1991) Tests of the roles of two diffusible substances in long term potentiation: evidencc for nitric oxide as a possible early retrograde messenger. Proc Natl Acad Sci USA 88:1 1285-11289.

Sacklor TC, Schwartz JH (1990) Sensitizing stimuli cause translocation of protein kinase $\mathrm{C}$ in Aplysia sensory neurons. Proc Natl Acad Sci USA 87:2036-2039.

Schacher S, Proshansky E (1983) Neurite regeneration by Aplysia neurons in dissociated cell culture: modulation by Aplysia hemolymph and the presence of the initial axonal segment. J Neurosci $3: 2403$ 2413.

Schacher S, Castellucci VF, Kandel ER (1988) cAMP evokes longterm facilitation in Aplysia sensory neurons that requires new protein synthesis. Science 240:1667-1669.

Schacher S, Montarolo P, Kandel ER (1990) Selective short- and longterm effects of serotonin, small cardioactive peptidc, and tetanic stimulation on sensorimotor synapses of Aplysia in culture. J Neurosci 10:3286-3294.

Schuman EM, Madison DVA (1991) A requirement for the intercellular messenger nitric oxide in long-term potentiation. Science 254 : $1503-1506$.

Seamon KB, Daly JW (1986) Forskolin: its biological and chemical properties. In: Advances in cyclic nucleotide and protein phosphorylation research (Greengard P. Robison GA, eds), pp 1-150. New York: Raven.

Silva AJ, Stevens CF, Tonegawa S, Wang Y (1992a) Deficient hippocampal long-term potentiation in $\alpha$-Calcium-Calmodulin Kinase II mutant mice. Science 257:201-206.

Silva AJ, Paylor R, Wehner JM, Tonegawa S (1992b) Impaired spatial learning in $\alpha$-Calcium-Calmodulin Kinase II mutant mice. Science 257:206-211.

Sugita S, Goldsmith JR, Baxter DA, Byrne JH (1992) Involvement of protein kinase $C$ in serotonin-induced spike broadening and synaptic facilitation in sensorimotor connections of Aplysia. J Neurophysiol 68:643-651.

Walters ET, Byrne JH (1983) Associative conditioning of single sensory neurons suggests a cellular mechanism for learning. Science 219 ; $405-408$. 